\title{
CONTRIBUCIÓN DE LAS COOPERATIVAS AGROALIMENTARIAS AL DESARROLLO SOSTENIBLE DE LAS EXPLOTACIONES DE SUS SOCIOS.
}

\author{
$\underline{\text { Jorge Luis Sánchez Navarro }}^{a}, \underline{\text { Narciso Arcas Lario }} * a^{\text {y Miguel Hernández Espallardo }}{ }^{b}$ \\ ${ }^{a}$ Universidad Politécnica de Cartagena (Cartagena, jorgel.sanchez@upct.es y arcas.lario@upct.es). \\ ${ }^{b}$ Universidad de Murcia, (Murcia,migher@um.es)
}

\section{Resumen}

Las exigencias de la sociedad están afectando a la capacidad de las empresas agrarias para competir. Cada vez más, se les exige cumplir con unas normas y requisitos relacionados con el desarrollo sostenible (protección del medio ambiente, bienestar animal, seguridad alimentaria...) que conllevan unos mayores costes de producción y comercialización. En este escenario, las cooperativas agroalimentarias se configuran como una alternativa para ayudar a que las explotaciones de sus socios reduzcan los costes del cumplimiento de estas normas y requisitos, facilitándoles información, formación y asesoramiento.

Con la información obtenida de una encuesta a socios y no socios de cooperativas, este trabajo tiene como objetivo contrastar la contribución de las cooperativas agroalimentarias al cumplimiento, por parte de las explotaciones de sus socios, de las normas y requisitos de desarrollo sostenible. Los resultados obtenidos evidencian que las cooperativas agroalimentarias mejoran la capacidad de los agricultores para cumplirlas, así como la rentabilidad de las explotaciones de sus socios en este sentido. Además, se muestra que la satisfacción económica de los socios con su cooperativa es mayor que la de no socios con su comercializador.

Palabras clave: cooperativas, desarrollo sostenible, satisfacción.

\section{Introducción y objetivos}

El desarrollo sostenible se define como "aquel que satisface las necesidades del presente sin comprometer la capacidad de las generaciones futuras para satisfacer las suyas" (Naciones Unidas, 1978; p. 59). Para lograrlo, es necesaria la integración de tres dimensiones: crecimiento económico y equidad (dimensión económica), conservación de los recursos naturales y del medio ambiente (dimensión ambiental) y desarrollo social (dimensión social) (Naciones Unidas, 2015). En este contexto, la actividad agraria se considera como clave para garantizarlo, ya que se encarga de promover ecosistemas saludables y apoyar la gestión sostenible de los recursos naturales, de forma que no se comprometa la capacidad de producción, presente y futura, del planeta (Gómez-Limón y Reig-Martínez, 2013).

Al objeto de garantizar la contribución de la actividad agraria al desarrollo sostenible, han ido apareciendo normativas y regulaciones sobre la seguridad alimentaria, la protección del medio ambiente y el bienestar animal, entre otras. Estas condicionan la actividad agraria, siendo los productores los que se ven afectados en mayor medida por estos condicionantes. A lo anterior, hay que añadir la complejidad y las dificultades que acompañan a la norma, lo que convierte su cumplimiento en un gran reto para el sector agroalimentario, y de forma especial para los pequeños agricultores.

El cumplimiento de las normas lleva asociados unos mayores costes de producción y comercialización. Entre ellos, destacan los administrativos (entrega de documentación a la Administración, envío de solicitudes...), los financieros o de inversión (en maquinaria y bienes de equipo, pago de tasas, acceso a financiación...), los de oportunidad (referidos al menor tiempo y recursos disponibles para la realización de otras actividades) y los operativos (relacionados con el cambio en la forma en que se deben hacer las cosas).

En la mayoría de los sectores productivos de una economía, el incremento en los costes de producción y comercialización se traslada en unos precios de venta mayores (Menghi et al., 2011). Sin embargo, lo anterior es más complicado en el sector agrario. Se trata de un sector en el que existen muchos pequeños productores y unos pocos compradores de gran dimensión (Brouwer et al., 2011), lo que, unido al carácter perecedero de los bienes que obtienen, hace que los productores agrarios tengan escaso o nulo poder de decisión sobre el precio de sus productos.

Ante esta situación, la incorporación de los productores a cooperativas agroalimentarias se plantea como una alternativa para superar los mencionados retos. Estas organizaciones pueden facilitar a sus socios información y asesoramiento sobre las normas y requisitos de desarrollo sostenible y, a través de los servicios de formación y suministro que ofrecen, pueden promover la realización eficiente de las prácticas agrícolas necesarias para cumplirlas. 
A partir de lo expuesto, el objetivo del trabajo es contrastar la contribución de las cooperativas agroalimentarias al cumplimiento, por parte de las explotaciones de sus socios, de las normas y requisitos de desarrollo sostenible. Concretamente, se pretende comprobar su contribución al conocimiento, la actitud y la capacidad para cumplir las normas. Así mismo, se analiza el papel que desempeñan estas organizaciones en relación al incremento en costes y pérdida de rentabilidad que supone dicho cumplimiento. Por último, se compara el nivel de satisfacción económica de agricultores socios de cooperativas con esta, respecto al de no socios con su comercializador habitual.

\section{Metodología}

La población objeto de estudio está formada por los productores agrarios de la Región de Murcia. Para abordar los objetivos del trabajo se ha recopilado información sobre las variables comentadas anteriormente. Estas han sido medidas mediante escalas Likert de 7 puntos, de forma que los entrevistados manifiestan su grado de acuerdo con la afirmación que se indica $(1=$ total desacuerdo; $7=$ total acuerdo $)$.

El procedimiento utilizado ha sido una encuesta presencial realizada a agricultores socios de cooperativas y no socios. Para ello, se contactó de forma aleatoria con agricultores de 22 municipios, pertenecientes a las distintas comarcas de la Región de Murcia. Tras la recogida de la información se han conseguido 162 cuestionarios válidos (97 corresponden a socios y 65 a no socios).

Para comprobar si existen diferencias entre socios y no socios de cooperativas, se han comparado los valores medios para cada grupo mediante un contraste ANOVA, tomando como criterio para afirmar que existen diferencias significativas entre ambos grupos un p-valor menor o igual a 0,10 (información que se recoge en la parte inferior de los gráficos que muestran los valores obtenidos para cada concepto objeto de estudio).

\section{Resultados}

A continuación, se presentan y describen los resultados obtenidos para cada aspecto sobre el que se pretende comprobar la contribución de las cooperativas.

\subsection{Conocimiento, actitud y capacidad para aplicar las normas y requisitos}

El Gráfico 1 revela un adecuado conocimiento por parte de los productores agrarios sobre las normas y requisitos de desarrollo sostenible (se observan valores superiores al punto medio 4), no observando diferencias entre socios y no socios.

Gráfico 1. Conocimiento, actitud y capacidad para aplicar las normas y requisitos

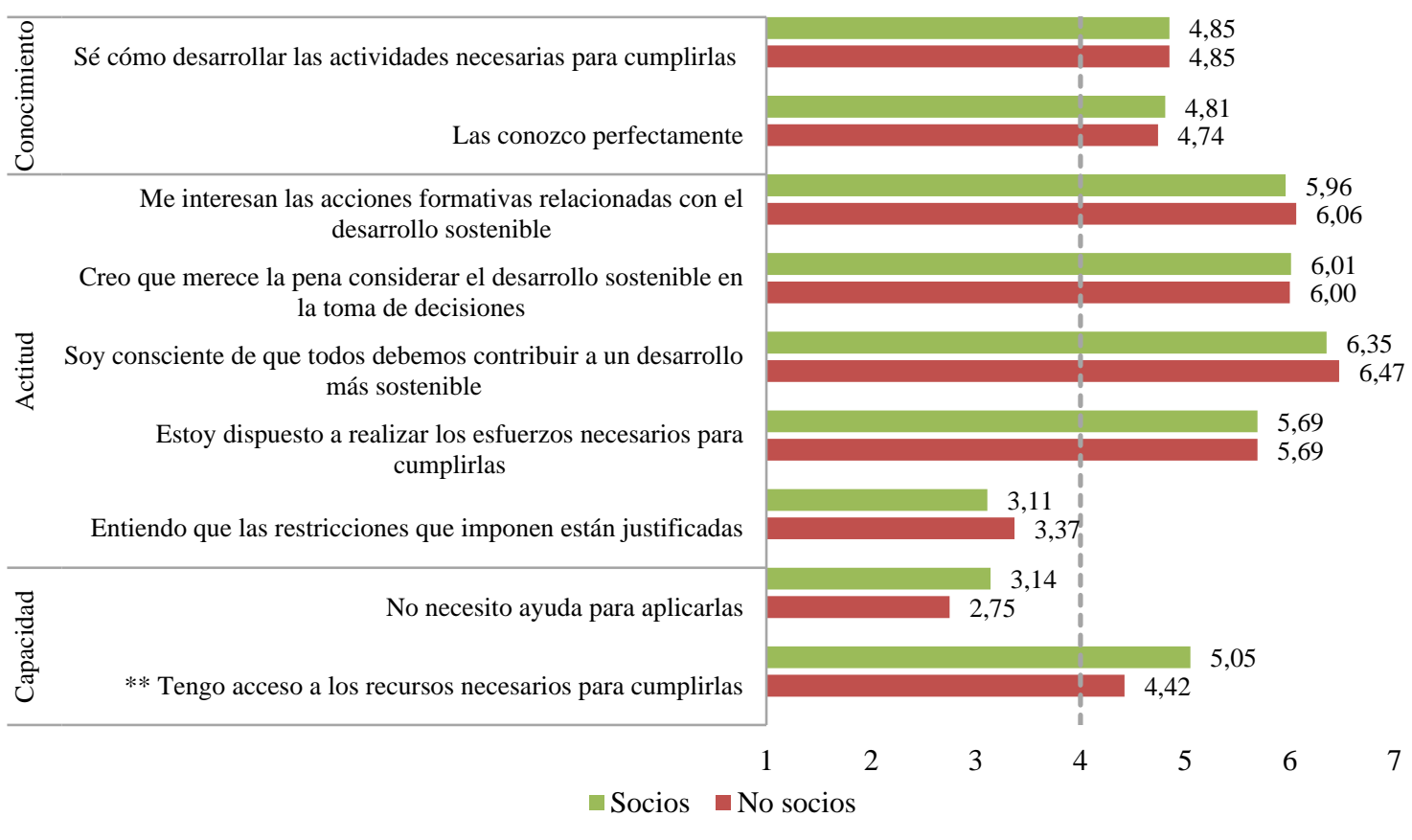

$*$ p-valor $<0,10 ; * *$ p-valor $<0,05 ; * * *$ p-valor $<0,01$ 
También se observa una buena actitud de los agricultores para cumplir las normas, ya que todos los ítems utilizados para medirla, excepto uno (entiendo que las restricciones que imponen están justificadas), presentan valores superiores a 4 . No se observan diferencias para el caso de socios y no socios.

Por último, respecto a la capacidad de los agricultores para cumplir las normas y requisitos se observa, por un lado, desacuerdo con la afirmación "No necesito ayuda para aplicarlas", lo que pone de manifiesto la necesidad de ayuda que precisan para cumplirlas y, por otro, la capacidad de acceso a los recursos necesarios para cumplirlas (superior al umbral 4), siendo esta mayor para el caso de agricultores socios que no socios.

\subsection{Costes y rentabilidad de cumplir las normas y requisitos}

El gráfico 2 muestra los resultados sobre las implicaciones que tiene el cumplimento de las normas y requisitos de desarrollo sostenible en el incremento de costes, y en la pérdida de rentabilidad de las explotaciones de agricultores socios y no socios.

En primer lugar, cabe destacar el elevado valor que presentan todos los ítems utilizados para medir los mayores costes, lo que implica que de forma mayoritaria los productores agrarios consideran que la producción y comercialización en base a las normas y requisitos de desarrollo sostenible conlleva unos mayores costes. Destacan, sobre el resto, los operativos, seguidos de los administrativos. Además, también se aprecia que dicho incremento en costes (administrativos, de oportunidad, de inversión y operativos) es menor para el caso de socios, aunque ninguna diferencia es estadísticamente significativa.

Respecto al impacto negativo que tienen las normas y requisitos de desarrollo sostenible en la rentabilidad de las explotaciones de los agricultores, se observa, de nuevo, que los valores encontrados son menores para el caso de agricultores socios, siendo además las diferencias estadísticamente significativas para dos de los ítems propuestos para medir tal aspecto.

Gráfico 2. Costes y rentabilidad de cumplir con las normas y requisitos de desarrollo sostenible

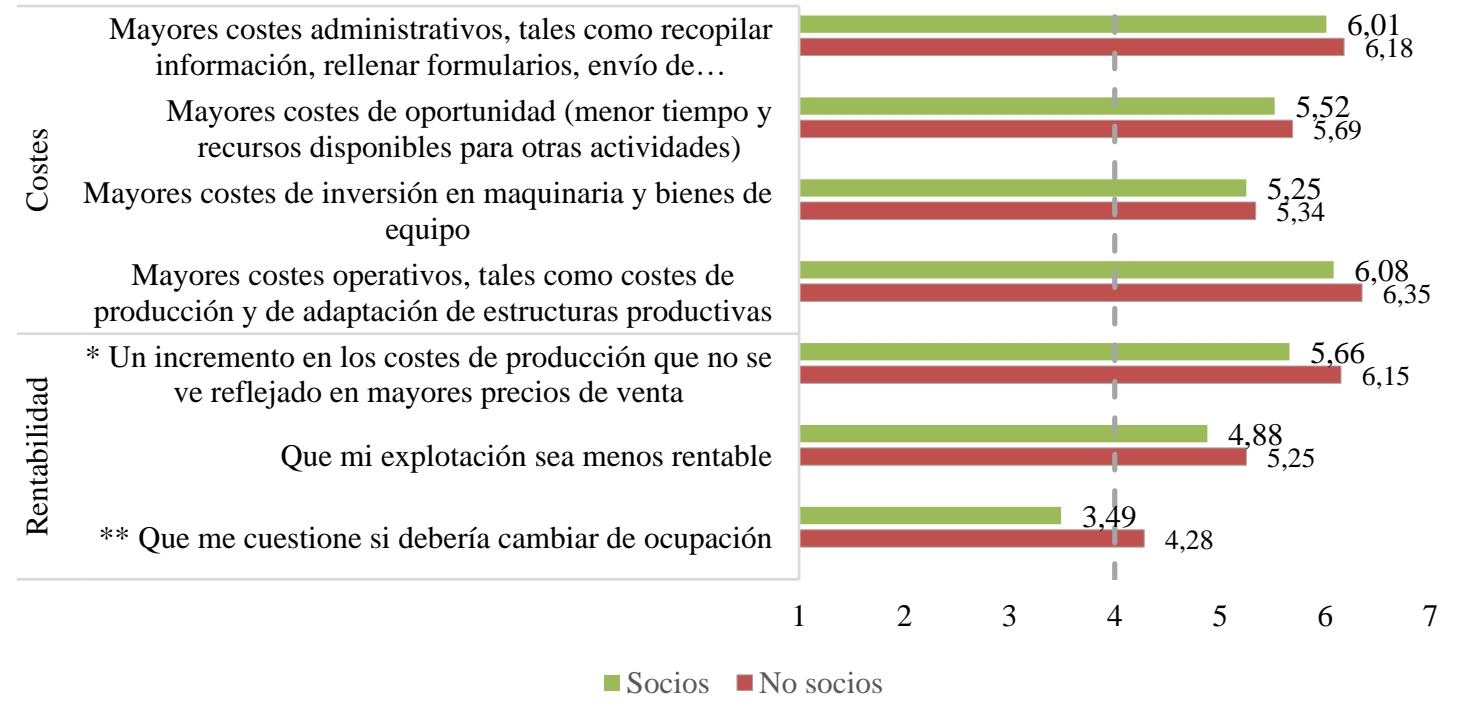

$*$ p-valor $<0,10 ; * *$ p-valor $<0,05 ; * * *$ p-valor $<0,01$

\subsection{Satisfacción económica}

Los resultados respecto a la satisfacción económica de los productores agrarios con su comercializador están claramente a favor de los socios de cooperativas (Gráfico 3) ya que, para todos los ítems propuestos para medirla, se observa que mientras que el valor para socios supera claramente el umbral de 4, para el caso de no socios no supera, e ningún caso, dicho umbral. Además, las diferencias encontradas son estadísticamente significativas. 
Gráfico 3. Satisfacción económica con el comercializador

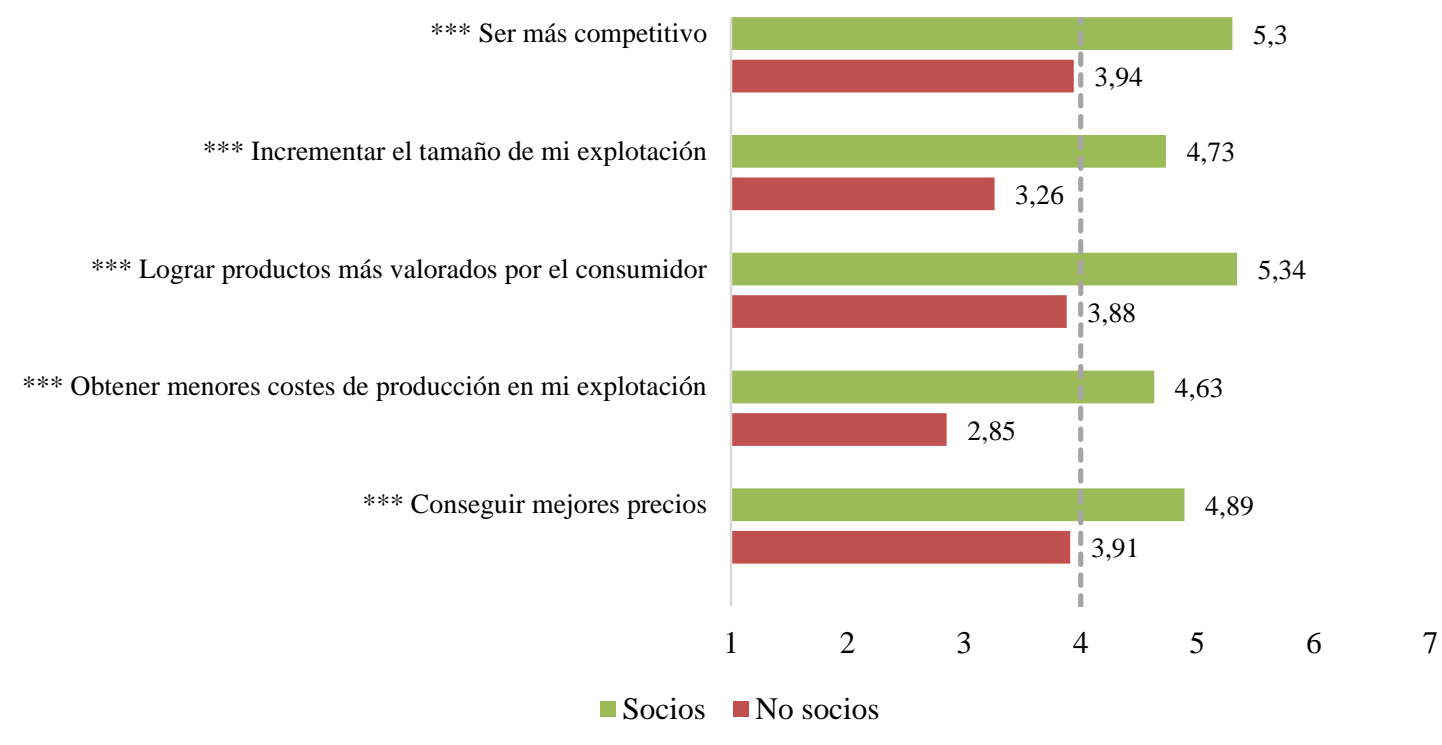

$*$ p-valor $<0,10 ; * *$ p-valor $<0,05 ; * * *$ p-valor $<0,01$

\section{Conclusiones}

Las cooperativas agroalimentarias pueden facilitar a sus socios información y asesoramiento sobre las normas y requisitos de desarrollo sostenible, así como las prácticas agrícolas necesarias para cumplirlas. Estas acciones ayudan a los productores socios a mejorar su actitud, con relación a estas normas y requisitos, y también sus habilidades para cumplirlas.

Además, los resultados obtenidos confirman que las cooperativas consiguen reducir la pérdida de la rentabilidad en las explotaciones de sus socios derivada del cumplimiento de las normas y requisitos de desarrollo sostenible. Ello puede obedecer a que estas organizaciones son capaces de suministrar insumos, equipos y maquinaria en condiciones más ventajosas. Además, las cooperativas pueden ayudar a sus socios a minimizar los costes del cumplimento de las referidas normas a través de la formación y el asesoramiento. Por último, en cuanto a la satisfacción de los productores agrarios con el comercializador elegido, los resultados revelan que los no socios de cooperativas están, en términos medios, insatisfechos con el comercializador elegido, mientras que los socios presentan un elevado nivel de satisfacción con su cooperativa. Se pone así de manifiesto la valoración positiva que los socios realizan del conjunto de la relación que mantienen con su cooperativa, con relación a la ayuda que reciben de ella para el cumplimento de las normas y requisitos sobre desarrollo sostenible.

Consideramos que los resultados obtenidos son de especial importancia para poner en valor la contribución de las cooperativas agroalimentarias al desarrollo sostenible, ya que, a su indudable aportación directa, se le añade la que hacen de forma indirecta, ayudando a que las explotaciones de sus socios afronten los retos relacionados con la sostenibilidad y, así, contribuyendo a ella.

\section{Bibliografía}

Naciones Unidas (1987). Asamblea General de las Naciones Unidad. Informe de la Comisión Mundial sobre el Medio Ambiente y el Desarrollo. En: https://undocs.org/es/A/42/427

Naciones Unidas (2015). Asamblea General de las Naciones Unidas Memoria del Secretario General sobre la labor de la Organización. En: https://undocs.org/es/A/70/1

Brouwer, F., Walker, A.J., Hoste, R. y Van Wagenberg, C. (2011). "Literature study on the cost of compliance with EU legislation in the fields of environment, food safety and animal welfare". European Commission D.G. Agriculture and Rural development.

Gómez-Limón, J. A. y Arriaza, M. (2011). Evaluación de la sostenibilidad de las explotaciones de olivar en Andalucía. Almería, España: Unicaja Fundación.

Menghi, A., De Roest, K., Parcelluzzi, A., Zazi, C., Wildegger, B., De Witte, T., ... Mettepenningen, E (2011). Assessing farmers' cost of compliance with EU legislation in the field of environmental, animal welfare and food safety. 